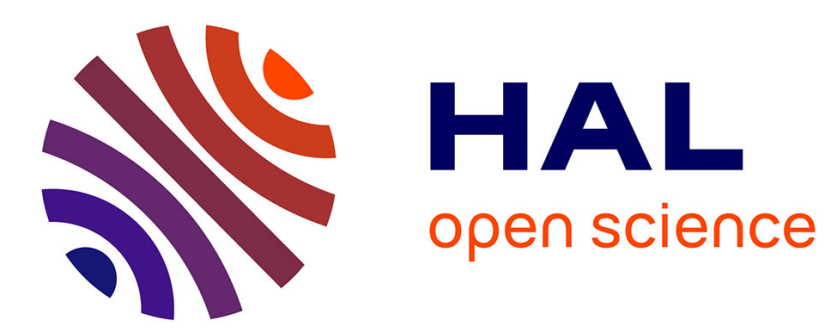

\title{
OR-PCA with MRF for Robust Foreground Detection in Highly Dynamic Backgrounds
}

Sajid Javed, Seon Ho Oh, Andrews Sobral, Thierry Bouwmans, Soon Ki Jung

\section{To cite this version:}

Sajid Javed, Seon Ho Oh, Andrews Sobral, Thierry Bouwmans, Soon Ki Jung. OR-PCA with MRF for Robust Foreground Detection in Highly Dynamic Backgrounds. Asian Conference on Computer Vision, ACCV 2014, Nov 2015, Singapore, Singapore. pp.284 - 299, 10.1007/978-3-319-16811-1_19 . hal-01374213

\section{HAL Id: hal-01374213 \\ https://hal.science/hal-01374213}

Submitted on 30 Sep 2016

HAL is a multi-disciplinary open access archive for the deposit and dissemination of scientific research documents, whether they are published or not. The documents may come from teaching and research institutions in France or abroad, or from public or private research centers.
L'archive ouverte pluridisciplinaire HAL, est destinée au dépôt et à la diffusion de documents scientifiques de niveau recherche, publiés ou non, émanant des établissements d'enseignement et de recherche français ou étrangers, des laboratoires publics ou privés. 


\title{
OR-PCA with MRF for Robust Foreground Detection in Highly Dynamic Backgrounds
}

\author{
Sajid Javed ${ }^{1}$, Seon Ho $\mathrm{Oh}^{1}$, Andrews Sobral ${ }^{2}$, \\ Thierry Bouwmans ${ }^{2}$ and Soon Ki Jung ${ }^{1}$ \\ ${ }^{1}$ School of Computer Science and Engineering, Kyungpook National University, \\ 80 Daehak-ro, Buk-gu,Daegu, 702-701, Republic of Korea \\ $\{$ sajid,shoh@vr.knu.ac.kr\},\{skjung@knu.ac.kr $\}$ \\ Laboratoire MIA (Mathematiques, Image et Applications)- Université de La \\ Rochelle, 17000, France, \{andrews.sobral, thierry.bouwmans@univ-lr.fr $\}$
}

\begin{abstract}
Accurate and efficient foreground detection is an important task in video surveillance system. The task becomes more critical when the background scene shows more variations, such as water surface, waving trees, varying illumination conditions, etc. Recently, Robust Principal Components Analysis (RPCA) shows a very nice framework for moving object detection. The background sequence is modeled by a low-dimensional subspace called low-rank matrix and sparse error constitutes the foreground objects. But RPCA presents the limitations of computational complexity and memory storage due to batch optimization methods, as a result it is difficult to apply for real-time system. To handle these challenges, this paper presents a robust foreground detection algorithm via Online Robust PCA (OR-PCA) using image decomposition along with continuous constraint such as Markov Random Field (MRF). OR-PCA with good initialization scheme using image decomposition approach improves the accuracy of foreground detection and the computation time as well. Moreover, solving MRF with graph-cuts exploits structural information using spatial neighborhood system and similarities to further improve the foreground segmentation in highly dynamic backgrounds. Experimental results on challenging datasets such as Wallflower, I2R, BMC 2012 and Change Detection 2014 dataset demonstrate that our proposed scheme significantly outperforms the state of the art approaches and works effectively on a wide range of complex background scenes.
\end{abstract}

\section{Introduction}

Foreground detection (also known as background subtraction) is one of the most important preprocessing step in many computer vision applications. Typically, the foreground detection process forms the first stage in automated visual surveillance systems, as well as other applications such as motion capture, object tracking and augmented reality.

Many algorithms have been developed to handle the problem of foreground detection in videos [1-3]. In recent years, Robust Principal Component Analysis 
(RPCA) based low-rank matrix decomposition algorithms have been used for foreground detection [4]. RPCA decomposes the original data matrix $A$ as a sum of low-dimensional subspace called low-rank matrix $L$ and correlated sparse outliers S. Fig. 1 shows an example of foreground detection using RPCA of original images taken from i-LIDS dataset [5].

As RPCA based approaches provide a nice framework for foreground detection, but it currently faces two major difficulties. Traditional RPCA based approaches use batch optimization, e.g. in order to decompose low-rank and sparse components, a number of samples are required to store. Therefore, it suffers from high memory cost and computational complexity.

In order to tackle these issues, this paper presents a robust foreground detection algorithm via Online Robust PCA (OR-PCA) on decomposed images from input image. We briefly explain our methodology here: First, input image is decomposed into Gaussian and Laplacian images. Then, OR-PCA is applied to each Gaussian and Laplacian images for background modeling. Since Gaussian image is robust against noise of small pixel variations and Laplacian image preserves edge features. Therefore, our methodology improves the quality of foreground as well as computational time using alternative initialization scheme in OR-PCA. Finally, an MRF is utilized to exploit structural information and similarities to improve the foreground segmentation.

The rest of this paper is organized as follows. In Section 2, the related work is reviewed. Section 3 describes our methodology in detail. Experimental results are discussed in Section 4. Finally, conclusions are drawn in Section 5.
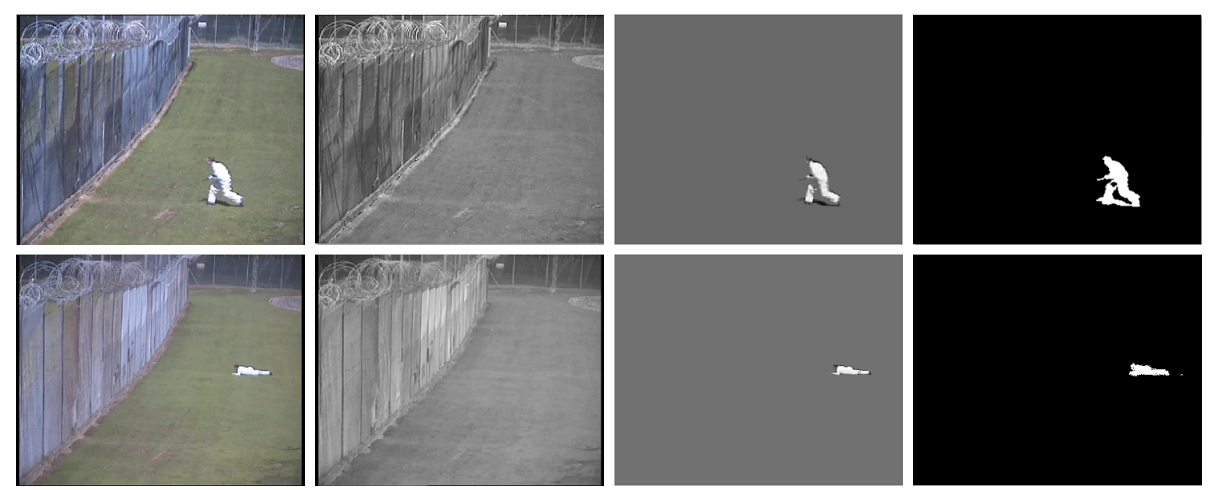

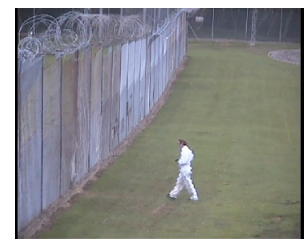

(a)

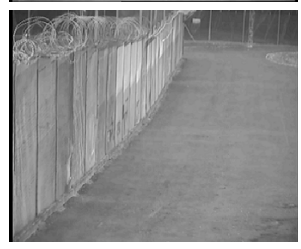

(b)

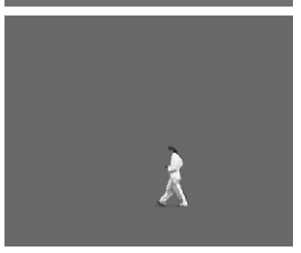

(c)

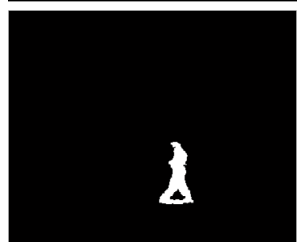

(d)

Fig. 1. An example of moving object detection using RPCA. From left to right: (a) input, (b) low-rank, (c) sparse component, and (d) foreground mask. 


\section{Related Work}

Over the past few years, excellent methods have been proposed for foreground object detection and tracking. Among them, RPCA [6] shows promising results for background modeling. Excellent surveys on background modeling using RPCA can be found in [4,7]. All these RPCA approaches, such as Augmented Lagrangian Multiplier (ALM), Singular Value Thresholding (SVT) and Linearized Alternating Direction Method with an Adaptive Penalty (LADMAP) discussed in [4], solve the sub-optimization problem in each iteration under defined convergence criteria in order to separate the low-rank matrix and sparse error. All these methods work in a batch optimization manner, as a result memory storage and time complexity problems occur. Therefore Principal Component Pursuit (PCP) via batch optimization is not acceptable for real-time system.

Many noticeable improvements have been found in the literature to accelerate the PCP algorithms [8]. For example, Zhou et al. [9] proposed Go Decomposition (GoDec) which accelerates RPCA algorithm via PCP using Bilateral Random Projections (BRP) scheme to separate the low-rank and sparse matrix. SemiSoft GoDec [9] method is an extension of GoDec which is four times faster than GoDec. It imposes hard thresholding scheme in low-rank and sparse matrix entries. In [10], Zhou et al. proposed Detecting Contiguous Outliers in the low-rank Representation (DECOLOR) method, which accelerates PCP algorithm by integrating the object detection and background learning into a single process of optimization. It also adds continuity constraints on low-rank and sparse components. In [11], a fast PCP algorithm is proposed, which reduces the SVD computational time in inexact ALM (IALM) by adding some constants in the minimization equation of low-rank and sparse. The results in background modeling case are very encouraging, but it is due to the base of PCP not desirable for real-time processing.

Incremental and online robust PCA methods are also developed for PCP algorithms. Fore example, in [12], He et al. proposed Grassmanian Robust Adaptive Subspace Tracking Algorithm (GRASTA), which is an incremental gradient descent method on Grassmannian manifold for solving the RPCA problem in online manner. In its each iteration, GRASTA uses the gradient of the updated augmented Lagrangian function after revealing a new sample to perform the gradient descent. Results are encouraging for background modeling, but no theoretic guarantee of the algorithm convergence for GRASTA is provided. Therefore, in [13], an online learning method for sparse coding and dictionary learning is proposed which efficiently solves the smooth non convex objective function over a convex set. A real-time processing is achieved, but it does not require learning rate tunning like regular stochastic gradient descents.

In [14], Guan et al. proposed non-negative matrix factorization (NMF) method which receives one chunk of samples per step and updates the basis accordingly. NMF converges faster in each step of basis update. But, using a buffering strategy both time complexity and space remain the issue for handling large datasets. Therefore, Feng and Xu [15] recently proposed Online Robust-PCA (OR-PCA) algorithm which processes one sample per time instance using stochastic approx- 
imations. In this approach, a nuclear norm objective function is reformulated and therefore all the samples are decoupled in optimization process for sparse error separation. In this work, we develop a background/foreground separation method based on the OR-PCA which is modified to be adapted for this application.

\section{$3 \quad$ Methodology}

In this section, we discuss our scheme for foreground detection in detail. Our methodology consists of several components which are described as a system diagram in Fig. 2.

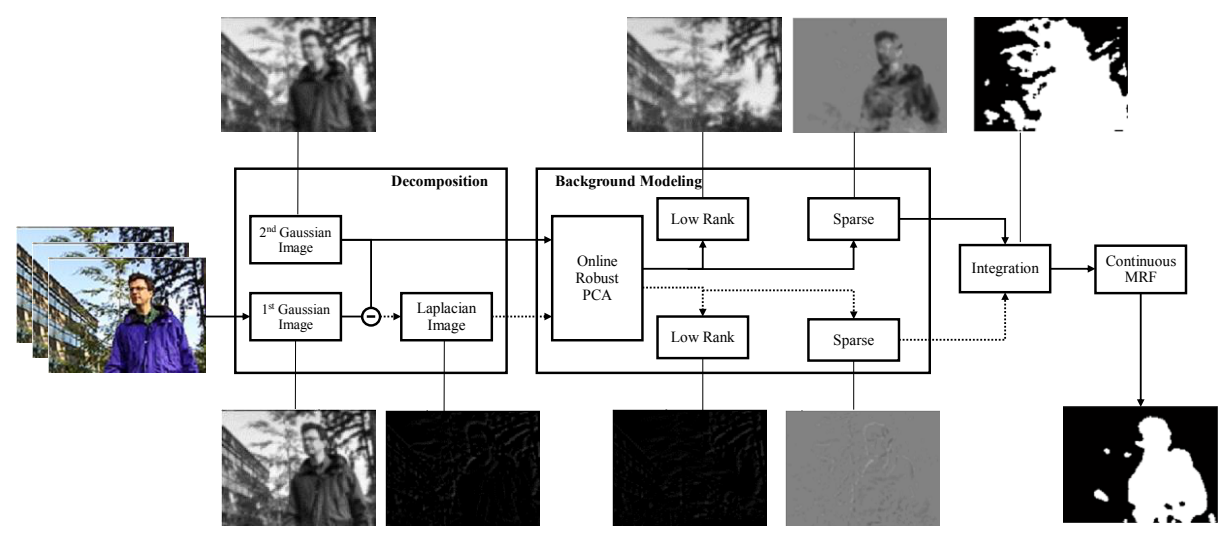

Fig. 2. Overview of our background modeling scheme.

Our methodology consists of four main stages: decomposition, background modeling, integration and continuous MRF. Initially, the input video frames are decomposed into Gaussian and Laplacian images using a set of two Gaussian kernels. Then, OR-PCA is applied to each of Gaussian and Laplacian images with different parameters to model the background, separately. In the background modeling stage, we have proposed an alternative initialization scheme to speed up the stochastic optimization process. Finally, the integration stage, which combines low-rank and sparse components obtained via OR-PCA to recover the background model and foreground image, is performed. The reconstructed sparse matrix is then thresholded to get the binary foreground mask. In order to improve the foreground segmentation, a MRF is applied which exploits structural information and similarities continuously. In the following sections, we will describe each module in detail.

\subsection{Decomposition}

In the first stage, two separate spatial Gaussian kernels are designed to decompose the input image into Gaussian and Laplacian images. First, Gaussian 
kernels are applied on the input image to get the Gaussian images. In the first case, we choose the standard deviation $\sigma$ on the Gaussian kernel as 2 with a filter size of $5 \times 5$ to get the first Gaussian image. In the second case, we apply Gaussian kernel with a same $\sigma$ value on the first blurred image due to its enough smoothing properties. Since the difference of Gaussians is approximately same as Laplacian of Gaussian, therefore Laplacian image is obtained by the difference of two Gaussian images.

Every input video frame is decomposed into Gaussian and Laplacian images using the method discussed above. As Gaussian image is robust against background variations and Laplacian image provides enough edge features for small pixels variations. Therefore, the false alarms are reduced from foreground region to some extent as a result, and our methodology provides accurate foreground detection.

\subsection{Background Modeling}

Online Robust PCA [15] is used to model the background from Gaussian and Laplacian images. OR-PCA decomposes the nuclear norm of the objective function of the traditional PCP algorithms into an explicit product of two low-rank matrices, i.e., basis and coefficient. Thus, OR-PCA can be formulated as

$$
\begin{aligned}
\min _{L \in \Re^{p \times n}, R \in \Re^{n \times r}, E} & \left\{\frac{1}{2}\left\|Z-L R^{T}-E\right\|_{F}^{2}\right. \\
+ & \left.\frac{\lambda_{1}}{2}\left(\|L\|_{F}^{2}+\|R\|_{F}^{2}\right)+\lambda_{2}\|E\|_{1}\right\},
\end{aligned}
$$

where $Z$ is an input data, $L$ is a basis, $R$ is a coefficient, and $E$ is a sparse error. $\lambda_{1}$ controls the basis and coefficients for low-rank matrix, whereas $\lambda_{2}$ controls the sparsity pattern, which can be tunned according to video analysis. In addition, basis and coefficient depend on the value of rank.

In particular, the OR-PCA optimization consists of two iterative updating components. Firstly, the input video frame is projected onto current initialized basis and we separate the sparse noise component, which includes the outliers contamination. Then, the basis is updated with a new input video frame. More details can be found in [15].

The background sequence for each image is then modeled by a multiple of basis $L$ and its coefficient $R$, whereas the sparse component $E$ for each image constitutes the foreground objects.

Initialization. The number of subspace basis is randomly determined using improper value of rank, and no initialization method is considered for OR-PCA in [15]. The rank value $R$ is 20 and $\lambda_{1}=\lambda_{2}=0.01$ in Eq.(1). As a result, the algorithm converges slowly to the optimal solution and outliers appear in the low-rank matrix, which effects the sparse component as well as foreground mask for background modeling case, as shown in Fig. 3. 


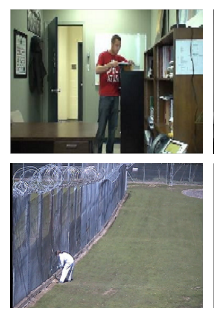

(a)

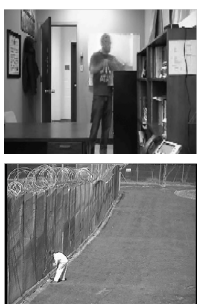

(b)

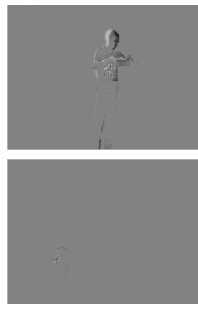

(c)

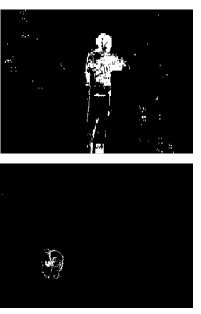

(d)

Fig. 3. OR-PCA failure using $r=20$ and $\lambda_{1}=\lambda_{2}=0.01$. From left to right: (a) input, (b) low-rank, (c) sparse component, and (d) foreground mask.

In order to meet the time complexity, the basis for low-dimensional subspace is initialized using first $N$ video frames with a good selection of rank. Since we are applying OR-PCA on two images in our scheme, the basis for each image is initialized according to this scheme. In this case, the rank is a tunable parameter for each image, that will be discussed in the later section.

By this technique, the OR-PCA converges to the low-dimensional subspace faster than original one. The outliers are also reduced and good computational time is achieved without sacrificing the quality of foreground in surveillance case.

\subsection{Integration}

The low-rank and sparse components are obtained from each decomposed image after applying OR-PCA. Gaussian and Laplacian low-rank and sparse components are integrated in this step. We use different parameters setting for OR-PCA in Eq.(1) on each decomposed image. $\lambda_{1}$ is considered as a constant 0.01 for both images. $\lambda_{2}$ and rank $r$ for Laplacian, whereas $\lambda_{2}^{\prime}$ and rank $r^{\prime}$ for Gaussian image are selected according to background scene, for obtaining enough sparsity pattern for each decomposed image.

Since Laplacian image provides enough edge features for small variations in background scene, therefore $\lambda_{2}$ must be smaller than $\lambda_{2}^{\prime}$. After integrating both components of each image, the binary foreground mask $f$ is then obtained by thresholding the integrated sparse component.

At this stage, the background subtraction scheme is good enough to deal with static and some small background dynamics such as slightly illumination changes, but it fails to handle highly dynamic backgrounds, where most part of the background pixels have high variations such as waving trees, water surface, rapid illumination changes, etc. For example, in Fig. 4 (a), (b) and (c) show the results of static and some small dynamic backgrounds. However, moving curtain and waving trees where most part of the background pixels are moving are shown in (d) and (e), respectively. We use the best parameters as $r=1$ and $\lambda_{2}=0.03$ for both images in (a). Whereas in (b) and (c), $r=1, r^{\prime}=3, \lambda_{2}=0.02$ and $\lambda_{2}^{\prime}=0.04$ are used for each decomposed images. Similarly, the best parameters are also considered for (d) and (e) as $r=1, r^{\prime}=10, \lambda_{2}=0.02$ and $\lambda_{2}^{\prime}=0.06$, respectively. 

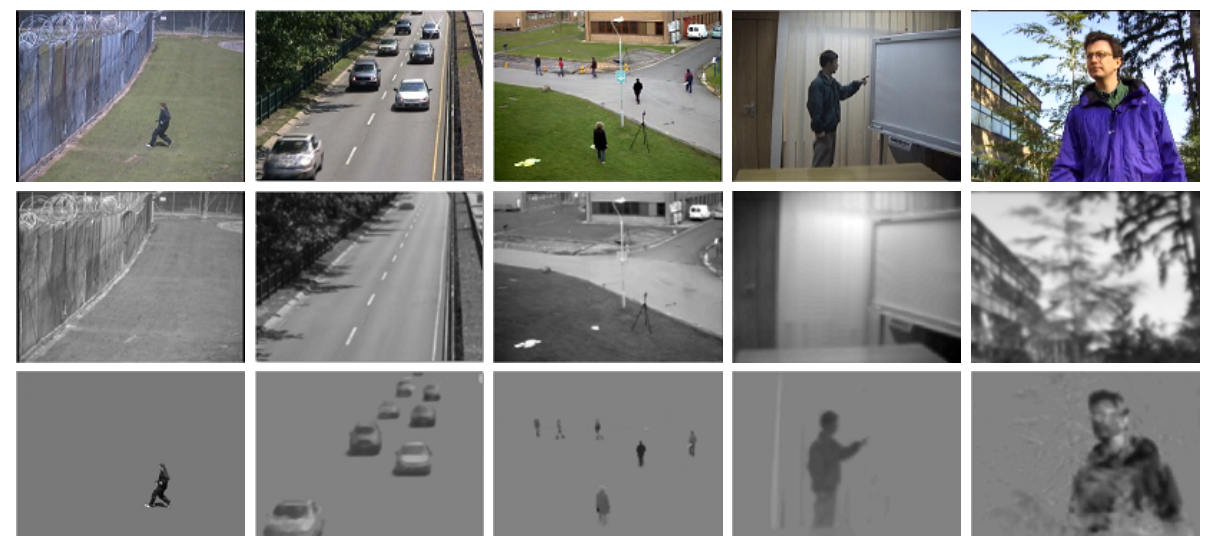

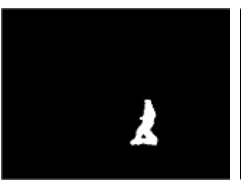

(a)

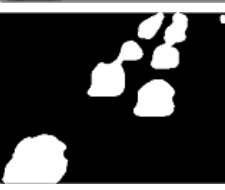

(b)

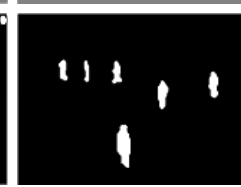

(c)

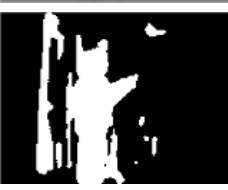

(d)

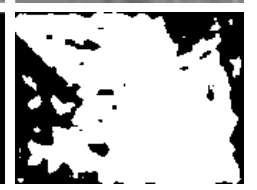

(e)

Fig. 4. OR-PCA via image decomposition. Input, low rank, sparse and foreground mask images are shown in each of rows.

OR-PCA on decomposed images without a continuous constraint is not robust to handle highly dynamic background scenes as mentioned above. As a result a large number of false alarms are generated, which is not useful for visual surveillance system. Therefore, we have employed a continuous constraint in the foreground mask such as MRF, which improves the quality of foreground segmentation in static as well as in highly dynamic backgrounds.

\subsection{Improving Foreground Segmentation with MRF}

The foreground labels can be not optimal, and therefore it can be improved with spatio-temporal constraints. In this paper, we utilize an MRF to optimize the labeling field. The MRF is a set of random variables having a Markov property described by an undirected graph.

Let us consider the foreground image $f$ as a set of pixels $\mathcal{P}$ and a set of labels $\mathcal{L}=\{0,1\}$, such that

$$
f_{p}= \begin{cases}0, & \text { if } p \text { belongs to background } \\ 1, & \text { if } p \text { belongs to foreground. }\end{cases}
$$

The goal is to find a labeling $f$ which minimizes the energy function:

$$
E(f)=\sum_{p \in P} D_{p}\left(f_{p}\right)+\sum_{p, q \in \mathcal{N}} V_{p, q}\left(f_{p}, f_{q}\right)
$$




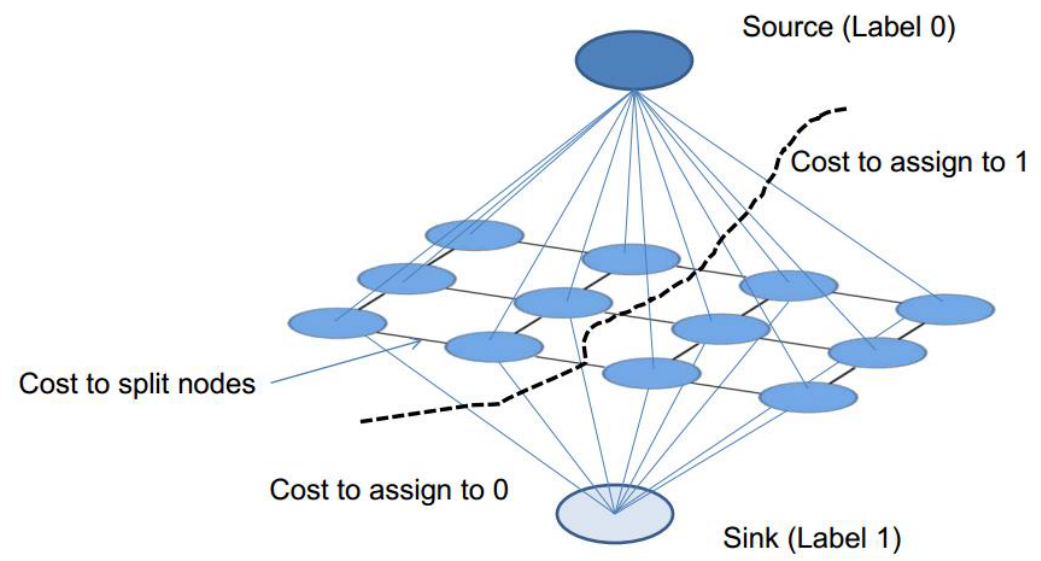

Fig. 5. Overview of solving MRF with graph cuts algorithm.

where $\mathcal{N} \subset \mathcal{P} \times \mathcal{P}$ is a neighborhood system on pixels. $D_{p}\left(f_{p}\right)$ is a function derived from the observed data that measures the cost of assigning the label $f_{p}$ to the pixel $p$. $V_{p, q}\left(f_{p}, f_{q}\right)$ measures the cost of assigning the labels $f_{p}, f_{q}$ to the adjacent pixels $p, q$. The energy functions like $E$ are extremely difficult to minimize, however, as they are nonconvex functions in a space with many thousands of dimensions. In the last few years, however, efficient algorithms have been developed for these problems based on graph cuts.

The basic idea of graph cuts is to construct a directed graph $\mathcal{G}=(\mathcal{V}, \mathcal{E})$, where the vertices $V$ stands for all pixels in image and edges $E$ denotes spatially neighboring pixels having nonnegative weights that has two special vertices (terminals), namely, the source $s$ and the sink $t$.

MRF that has such type of $s-t$ graph is called graph-representable as shown in Fig. 5 and can be solved in polynomial time using graph cuts [16].

In this work, we have used the gco-v3.0 library ${ }^{1}$ for optimizing multi-label energies via the $\alpha$-expansion and $\alpha$ - $\beta$-swap algorithms. It supports energies with any combination of unary, pairwise, and label cost terms.

\section{Experimental Results}

In this section we present a set of both qualitative and quantitative experiments on four challenging datasets namely Change Detection ${ }^{2} 2014$ [17], Wallflower ${ }^{3}$ [18], $\mathrm{I}^{2} \mathrm{R}^{4}[19]$ and Background Models Challenge ${ }^{5}$ (BMC) 2012 [20] dataset.

\footnotetext{
${ }^{1}$ http://vision.csd.uwo.ca/code/

${ }^{2}$ http://www.changedetection.net/

${ }^{3}$ http://research.microsoft.com/en-us/um/ people/jckrumm/wallflower/testimages.htm

${ }^{4}$ http://perception.i2r.a-star.edu.sg/bk_model/bk_index.html

${ }^{5}$ http://bmc.univ-bpclermont.fr/
} 
We compare our method with other well known state of the art methods, e.g., Mixture of Gaussians (MOG) [21], Semi Soft GoDec [9] and DECOLOR [10]. First we describe our experimental settings and then we analyzed the results obtained from different datasets in detail.

\subsection{Experimental Settings}

As our algorithm is based on two frames processing, therefore different parameters setting are considered for each frame in Eq.(1). We use same parameters as described in Fig. 4. Here we describe the range for each category. In case of static backgrounds, we use rank as 1 and $\lambda_{2} \in(0.01,0.04]$ for both images. However, in case of highly dynamic backgrounds such as waving trees, water surface, fountain, etc, the rank $r$ must be in the range of $r \in(1,3]$ and $\lambda_{2} \in(0.01,0.03]$ for Laplacian image, whereas the rank $r^{\prime} \in(r, 8]$ and $\lambda_{2}^{\prime} \in\left(\lambda_{2}, 0.09\right]$ for Gaussian image, respectively. Similarly, for Bootstraping case, the rank $r \in(1,5]$ and $\lambda_{2} \in(0.01,0.05)$ for Laplacian image and rank $r^{\prime} \in(5,10)$ and $\lambda_{2}^{\prime} \in(0.03,0.06]$ for Gaussian image.

\subsection{Qualitative Analysis}

Qualitative results are presented on selected video sequences from each dataset. Our algorithm is implemented in Matlab R2013a with $3.40 \mathrm{GHz}$ Intel core i5 processor with 4 GB RAM. Additionally, $5 \times 5$ median filtering is applied as a post-processing step on binary foreground mask.

Change Detection 2014 Dataset. From Change Detection 2014 dataset [17], five sequences namely, office and pedestrains from category baseline, whereas canoe, fountain 2 and overpass from category dynamic backgrounds are selected. The image size of each sequence is $320 \times 240$. Fig. 6 shows the visual results of change detection dataset.

I2R Dataset. Five sequences namely, moving curtain, water surface, fountain, lobby and hall sequences are selected out of nine from I2R dataset [19]. Each sequence contains a $160 \times 128$ of frame size. Fig. 7 shows results of I2R dataset.

Wallflower Dataset. Four sequences namely, waving trees, camouflage, foreground aperture (FA) and light switch (LS) sequences are taken out of seven from Wallflower dataset [18]. Each frame contains a frame size of $160 \times 120$. Fig. 8 shows the qualitative results of wallflower dataset.

Background Models Challenge Dataset. Six sequences namely, Video 001, Video 002, Video 004, Video 005, Video 006 and Video 007 are tested out of nine from BMC 2012 dataset [20]. Each video sequence contains a frame size of $320 \times 240$. Fig. 9 shows the qualitative results of BMC dataset with other state-of-the-art methods. 


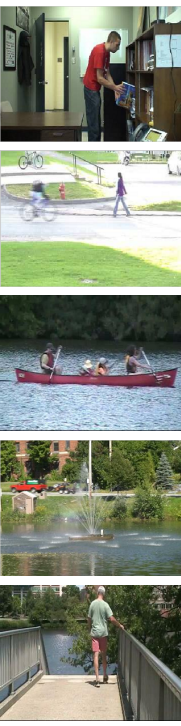

(a)

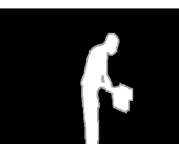

$\$$
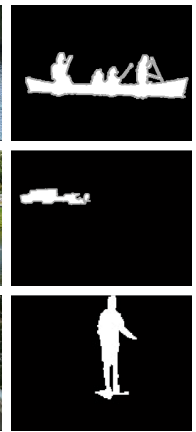

(b)

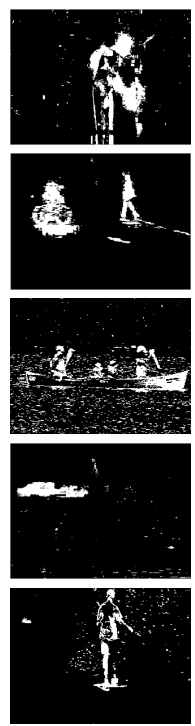

(c)

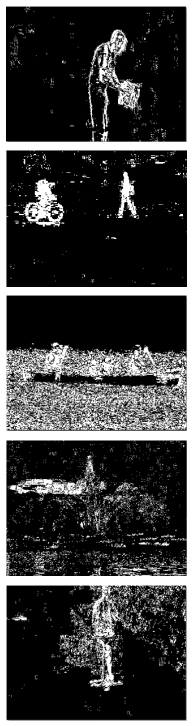

(d)

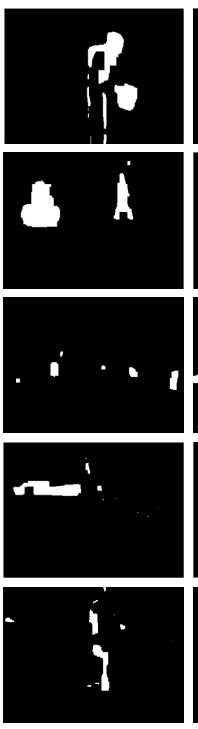

(e)
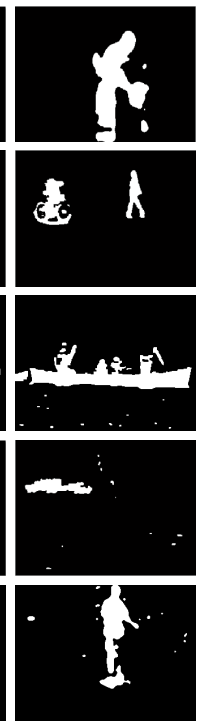

(f)

Fig. 6. Qualitative comparisons of Change Detection Dataset. From left to right: (a) input, (b) ground truth, (c) MOG, (d) Semi Soft GoDec, (e) DECOLOR, and (f) our method.

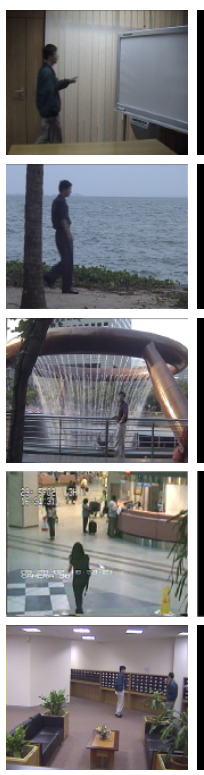

(a)
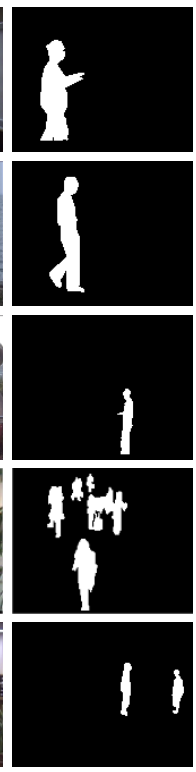

(b)
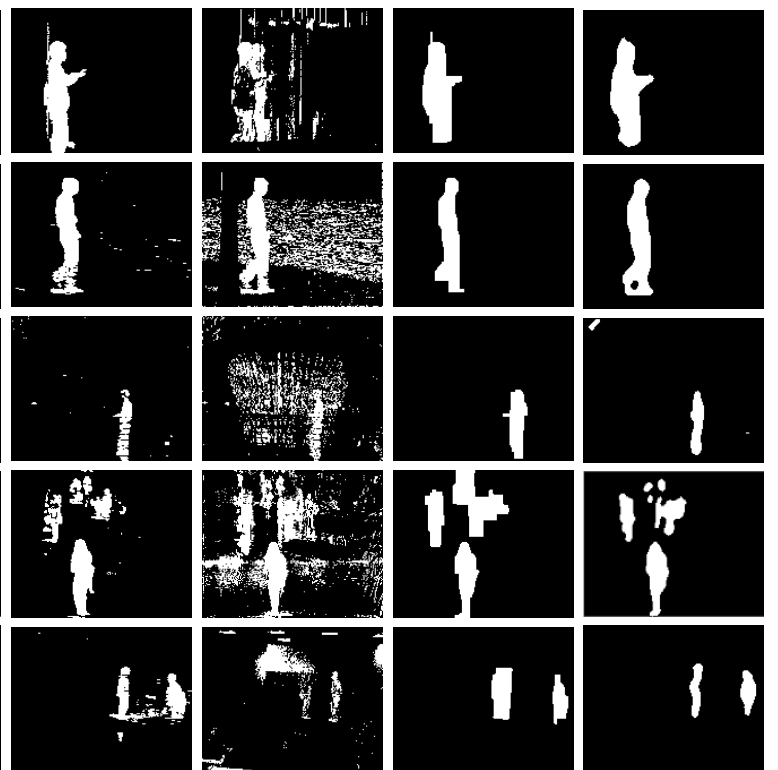

(c)

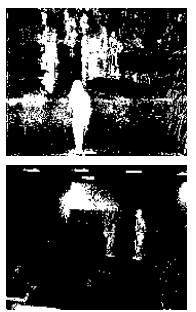

(d)

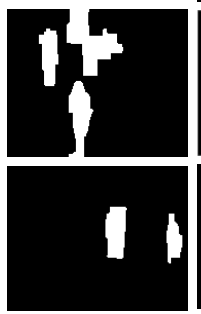

(e)

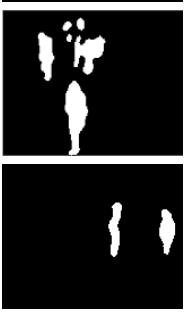

(f)

Fig. 7. Qualitative comparisons of I2R Dataset. From left to right: (a) input, (b) ground truth, (c) MOG, (d) Semi Soft GoDec, (e) DECOLOR, and (f) our method. 


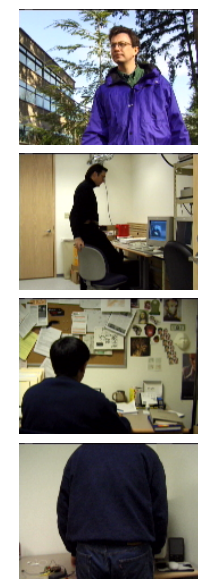

(a)

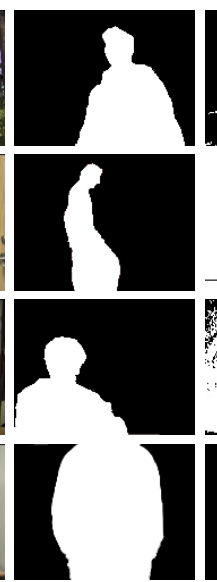

(b)

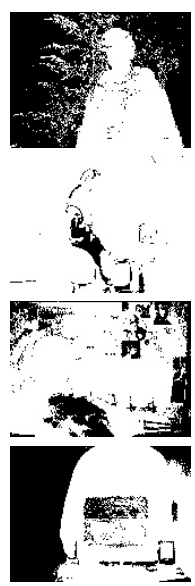

(c)

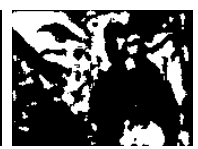

(n) n.:

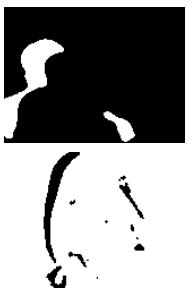

(d)

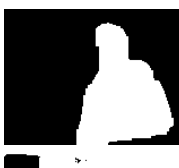

$\because$

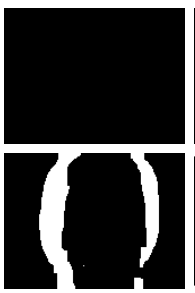

(e)
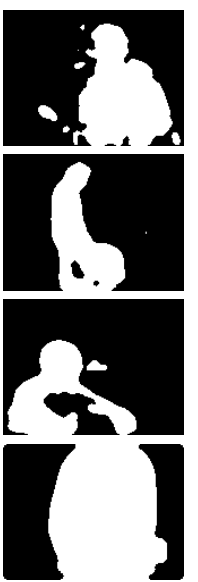

(f)

Fig. 8. Qualitative comparisons of Wallflower Dataset. From left to right: (a) input, (b) ground truth, (c) MOG, (d) Semi Soft GoDec, (e) DECOLOR, and (f) our method.

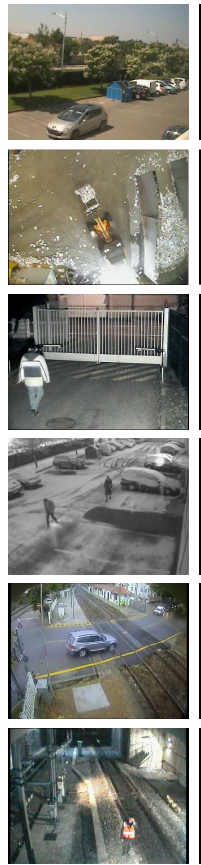

(a)
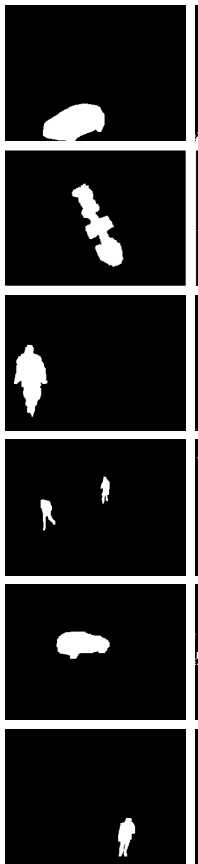

(b)
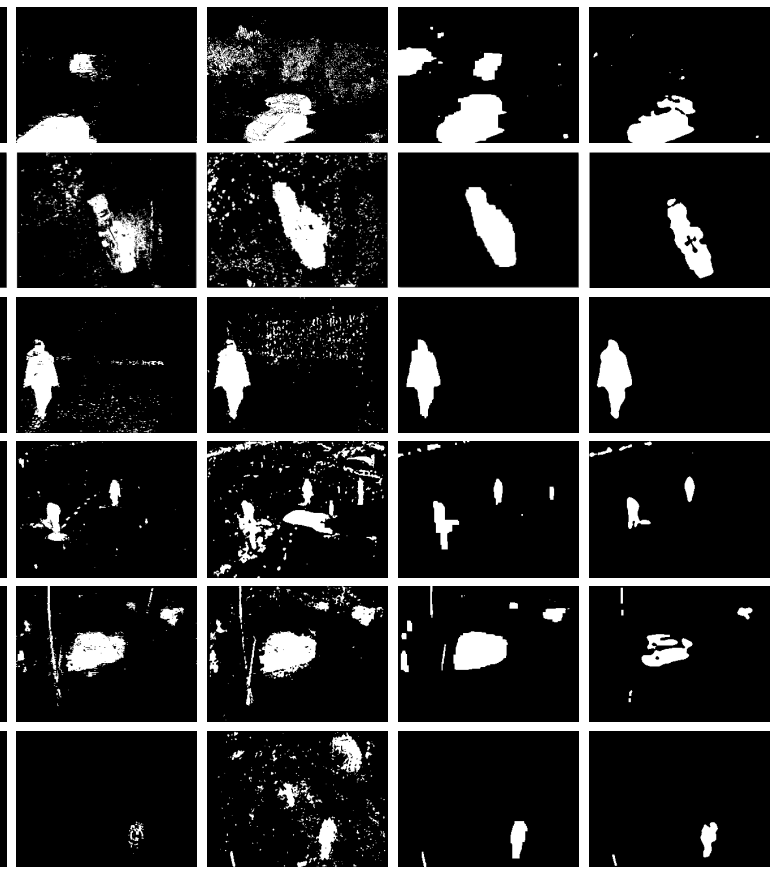

(e)

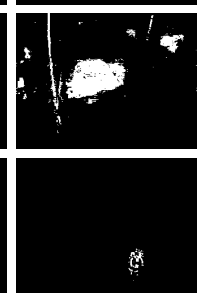

(c)

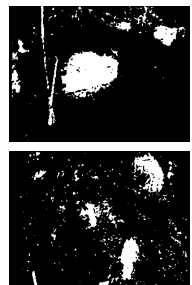

(d)

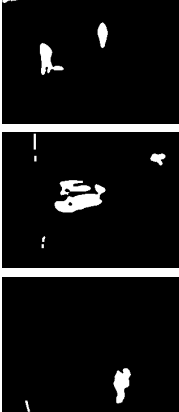

(f)

Fig. 9. Qualitative comparisons of BMC 2012 Dataset. From left to right: (a) input, (b) ground truth, (c) MOG, (d) Semi Soft GoDec, (e) DECOLOR, and (f) our method.

\subsection{Quantitative Analysis}

We also evaluate our algorithm quantitatively with other methods. F-measure score is computed for all sequences, by comparing our results with their available 
corresponding ground truth data. The $F$-measure is given as

$$
F_{\text {measure }}=\frac{2 \times \text { Recall } \times \text { Precision }}{\text { Recall }+ \text { Precision }},
$$

where Recall and Precision are computed based on true positives, false positives and false negatives respectively. Wallflower, Change Detection and I2R datasets are quantitatively evaluated according to this criteria. But BMC 2012 is evaluated according to their provided procedural tool ${ }^{6}$.

Table 1. Change Detection Dataset: Comparison of F-measure score in \% (direct oneto-one correspondence with Fig. 6).

\begin{tabular}{|c|c|c|c|c|c|c|}
\hline Method & Office & Pedestrians & Canoe & Fountain2 & Overpass & Avg. \\
\hline MOG & 60.48 & 54.56 & 51.14 & 79.68 & 50.95 & 59.36 \\
\hline SemiSoft GoDec & 56.02 & 70.31 & 30.91 & 31.38 & 55.17 & 48.75 \\
\hline DECOLOR & 57.30 & 78.93 & 16.03 & 82.41 & 35.73 & 54.08 \\
\hline Ours & $\mathbf{8 8 . 3 0}$ & $\mathbf{8 6 . 4 3}$ & $\mathbf{8 5 . 3 4}$ & $\mathbf{8 5 . 1 7}$ & $\mathbf{8 2 . 7 2}$ & $\mathbf{8 5 . 5 9}$ \\
\hline
\end{tabular}

Table 2. I2R Dataset: Comparison of F-measure score in \% (direct one-to-one correspondence with Fig. 7).

\begin{tabular}{|c|c|c|c|c|c|c|}
\hline Method & Curtain & Water Surface & Fountain & Hall & Lobby & Avg. \\
\hline MOG & 77.09 & 77.23 & 77.66 & 68.02 & 58.98 & 71.79 \\
\hline SemiSoft GoDec & 43.44 & 44.73 & 25.74 & 57.13 & 36.02 & 41.45 \\
\hline DECOLOR & 87.00 & 90.22 & 20.75 & $\mathbf{8 1 . 6 9}$ & 64.60 & 68.85 \\
\hline Ours & $\mathbf{8 9 . 2 0}$ & $\mathbf{9 1 . 6 6}$ & $\mathbf{8 2 . 8 3}$ & 78.44 & $\mathbf{8 0 . 8 1}$ & $\mathbf{8 4 . 5 8}$ \\
\hline
\end{tabular}

Table 3. Wallflower Dataset: Comparison of F-measure score in \% (direct one-to-one correspondence with Fig. 8).

\begin{tabular}{|c|c|c|c|c|c|}
\hline Method & Waving Trees & LS & FA & Camouflage & Avg. \\
\hline MOG & 66.39 & 16.86 & 32.91 & 74.21 & 47.59 \\
\hline SemiSoft GoDec & 18.29 & 26.71 & 24.51 & 66.31 & 33.95 \\
\hline DECOLOR & $\mathbf{8 8 . 4 5}$ & - & - & 38.56 & 31.00 \\
\hline Ours & 86.89 & $\mathbf{8 5 . 1 7}$ & $\mathbf{6 9 . 1 0}$ & $\mathbf{9 1 . 1 8}$ & $\mathbf{8 3 . 0 8}$ \\
\hline
\end{tabular}

Table 1, 2, 3 and 4 show the achieved performance on each dataset. In each case, our algorithm outperforms with other state of art methods, on average F-measure of $85.59 \%, 84.58 \%, 83.08 \%$ and $86.19 \%$ in each dataset, respectively.

\footnotetext{
${ }^{6}$ http://bmc.univ-bpclermont.fr/?q=node $/ 7$
} 
Table 4. BMC Dataset: Comparison of PSNR/F-measure score in \% (direct one-to-one correspondence with Fig. 9)

\begin{tabular}{|c|c|c|c|c|}
\hline Video & MOG & SemiSoft GoDec & DECOLOR & Ours \\
\hline 1 & $24.71 / 69.45$ & $22.78 / 78.65$ & $24.32 / 70.20$ & $\mathbf{3 5 . 0 6} / \mathbf{8 9 . 1 6}$ \\
\hline 2 & $25.98 / 75.57$ & $23.64 / 80.35$ & $33.70 / 84.67$ & $\mathbf{3 4 . 1 1} / \mathbf{8 6 . 1 8}$ \\
\hline 4 & $37.44 / 90.65$ & $33.11 / 87.55$ & $\mathbf{4 6 . 3 5} / \mathbf{9 5 . 5 2}$ & $44.15 / 94.68$ \\
\hline 5 & $35.10 / 73.42$ & $21.42 / 69.54$ & $32.20 / 75.81$ & $\mathbf{4 0 . 3 8} / \mathbf{7 9 . 1 7}$ \\
\hline 6 & $27.28 / 77.93$ & $24.16 / 75.50$ & $\mathbf{2 8 . 1 2} / \mathbf{7 8 . 8 9}$ & $32.69 / 76.80$ \\
\hline 7 & $43.67 / 76.10$ & $26.19 / 72.27$ & $\mathbf{5 3 . 7 1} / \mathbf{9 3 . 5 1}$ & $51.69 / 91.19$ \\
\hline \hline Avg. & $32.36 / 77.18$ & $25.21 / 77.31$ & $36.40 / 83.10$ & $\mathbf{3 9 . 6 8} / \mathbf{8 6 . 1 9}$ \\
\hline
\end{tabular}

Time Complexity. The computational time is also investigated during our experiments. The computational time is recorded frame by frame in seconds and the average time of first hundred frames is computed of different frame size. In our method, time is proportional to the value of rank. Table 5 shows the comparison of computational time.

Time is computed for each case according to our experimental settings discussed above. Since traditional RPCA via PCP based algorithms, either gets fail to load large amount of input video frames or take longer time for optimization which is not useful for real time processing. However in our approach, we have achieved a real time processing using initialization scheme with OR-PCA, moreover image decomposition together with continuous contraint improves the quality of foreground. These good experimental both qualitative and quantitative evaluations are the consequences of our proposed OR-PCA based scheme.

Discussion. We also apply OR-PCA including MRF on input images to compare our performance with decomposed images. As discussed above, decomposed images accurately detect the foreground mask and increase the $F$-measure score. Therefore we visually analyze some sequences such as WT and pedestrians as shown in Fig. 10 to show that OR-PCA using decomposed images along with MRF provides good segmentation results and best $F$-measure as compare to apply it on input images. In each case, our algroithm gives best $F$-measure score, e.g., $95.65 \%, 86.89 \%$ and $96.25 \%$, respectively, whereas $78.65 \%, 79.10 \%$, and $89.50 \%$ are observed in case of input image.

Table 5. Comparison of computational time in seconds.

\begin{tabular}{|c|c|c|c|}
\hline Cases & Frame Size & OR-PCA & Ours \\
\hline \multirow{2}{*}{ Static } & $576 \times 720$ & 0.5120 & $\mathbf{0 . 0 5 1 2}$ \\
\cline { 2 - 4 } & $288 \times 360$ & 0.1226 & $\mathbf{0 . 0 1 4 0}$ \\
\hline \multirow{2}{*}{ Dynamic } & $240 \times 320$ & 0.130 & $\mathbf{0 . 0 2 8 1}$ \\
\cline { 2 - 4 } & $120 \times 160$ & 0.0260 & $\mathbf{0 . 0 0 7 4}$ \\
\hline \multirow{2}{*}{ Bootstrap } & $256 \times 320$ & 0.1166 & $\mathbf{0 . 0 4 6 8}$ \\
\cline { 2 - 4 } & $128 \times 160$ & 0.0213 & $\mathbf{0 . 0 1 4 9}$ \\
\hline
\end{tabular}




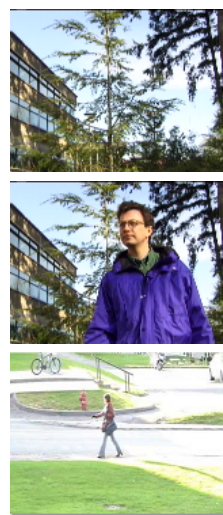

(a)
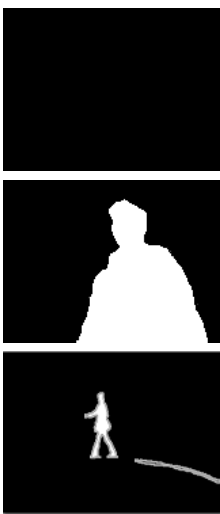

(b)

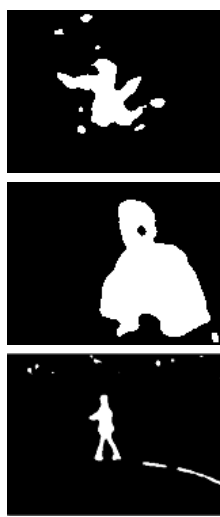

(c)

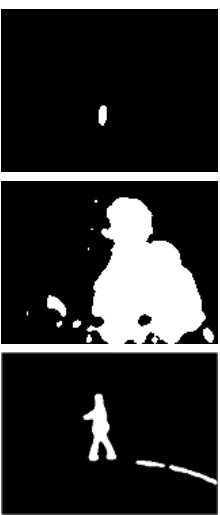

(d)

Fig. 10. From left to right: (a) input, (b) groundtruth, (c) OR-PCA with MRF on input image, and (d) ORPCA with MRF on decomposed images.

\section{Conclusion}

In this paper, a robust background modeling method against challenging background scenes is presented using OR-PCA via image decomposition with continuous MRF. Our methodology is robust against different background dynamics. We first decompose the input image then OR-PCA with initialization scheme including continuous constraint is applied with parameters tuning. As a result, computational complexity is reduced as compare to other PCP methods, and foreground segmentation is improved significantly, due to spatial Gaussian kernels and structural information in MRF. Experimental evaluations and comparisons with other state of the art methods show the effectiveness and robustness of our proposed scheme.

However, we just applied OR-PCA on two decomposed images and parameters are tuned manually. Therefore, our future work will focus more on brief analysis of each layer of hierarchical image decomposition with dynamical parameters setting, which adapts changes according to background dynamics.

\section{Acknowledgments}

This work is supported by the World Class 300 project, Development of HD video/network-based video surveillance system(10040370), funded by the Ministry of Trade, Industry, and Energy (MOTIE), Korea.

\section{References}

1. Bouwmans, T., El Baf, F., Vachon, B., et al.: Statistical background modeling for foreground detection: A survey. Handbook of Pattern Recognition and Computer Vision (2010) 181-199 
2. Bouwmans, T.: Traditional and recent approaches in background modeling for foreground detection: An overview. Computer Science Review (2014) $31-66$

3. Oh, S.H., Javed, S., Jung, S.K.: Foreground object detection and tracking for visual surveillance system: A hybrid approach. In: Frontiers of Information Technology (FIT), 2013 11th International Conference on. (2013) 13-18

4. Bouwmans, T., Zahzah, E.H.: Robust PCA via Principal Component Pursuit: A review for a comparative evaluation in video surveillance. Computer Vision and Image Understanding (2014) 22-34

5. Branch, H.O.S.D.: Imagery library for intelligent detection systems I-LIDS. In: Crime and Security, 2006. The Institution of Engineering and Technology Conference on. (2006) 445-448

6. Candès, E.J., Li, X., Ma, Y., Wright, J.: Robust Principal Component Analysis? Journal of the ACM (JACM) 58 (2011) 11-37

7. C. Guyon, T.B., Zahzah, E.H.: Robust principal component analysis for background subtraction: Systematic evaluation and comparative analysis. (2012) 223228

8. Javed, S., Oh, S.H., Heo, J., Jung, S.K.: Robust Background Subtraction via Online Robust PCA using image decomposition. In: Proceedings of the 2014 Research in Adaptive and Convergent Systems. (2014) 90-96

9. Zhou, T., Tao, D.: Godec: Randomized low-rank \& sparse matrix decomposition in noisy case. In: Proceedings of the 28th International Conference on Machine Learning (ICML-11). (2011) 33-40

10. Zhou, X., Yang, C., Yu, W.: Moving object detection by detecting contiguous outliers in the low-rank representation. Pattern Analysis and Machine Intelligence, IEEE Transactions on 35 (2013) 597-610

11. Rodriguez, P., Wohlberg, B.: Fast principal component pursuit via alternating minimization. In: Image Processing (ICIP), 20th IEEE International Conference on. (2013) 69-73

12. He, J., Balzano, L., Lui, J.: Online robust subspace tracking from partial information. [Online]. Available: http://arxiv.org/abs/1109.3827 (2011)

13. Mairal, J., Bach, F., Ponce, J., Sapiro, G.: Online learning for matrix factorization and sparse coding. The Journal of Machine Learning Research 11 (2010) 19-60

14. Guan, N., Tao, D., Luo, Z., Yuan, B.: Online nonnegative matrix factorization with robust stochastic approximation. Neural Networks and Learning Systems, IEEE Transactions on 23 (2012) 1087-1099

15. Feng, J., Xu, H., Yan, S.: Online robust PCA via stochastic optimization. In: Advances in Neural Information Processing Systems. (2013) 404-412

16. Kolmogorov, V., Zabin, R.: What energy functions can be minimized via graph cuts? Pattern Analysis and Machine Intelligence, IEEE Transactions on 26 (2004) 147-159

17. Goyette, N., Jodoin, P., Porikli, F., Konrad, J., Ishwar, P.: Changedetection.net: A new change detection benchmark dataset. In: Computer Vision and Pattern Recognition Workshops (CVPRW), IEEE Computer Society Conference on. (2012) $1-8$

18. Toyama, K., Krumm, J., Brumitt, B., Meyers, B.: Wallflower: principles and practice of background maintenance. In: Computer Vision, The Proceedings of the Seventh IEEE International Conference on. (1999) 255-261

19. Li, L., Huang, W., Gu, I.H., Tian, Q.: Statistical modeling of complex backgrounds for foreground object detection. Image Processing, IEEE Transactions on 13 (2004) $1459-1472$ 
20. Vacavant, A., Chateau, T., Wilhelm, A., Lequièvre, L.: A benchmark dataset for outdoor foreground/background extraction. In: Computer Vision-ACCV 2012 Workshops, Springer (2013) 291-300

21. Stauffer, C., Grimson, W.E.L.: Adaptive background mixture models for real-time tracking. In: Computer Vision and Pattern Recognition, IEEE Computer Society Conference on. Volume 2., IEEE (1999) 